\title{
A Bayesian Approach for Affine Auto-calibration
}

\author{
S.S. Brandt and K. Palander \\ Helsinki University of Technology, \\ Laboratory of Computational Engineering, \\ P.O. Box 9203, FI-02015 TKK, Finland
}

\begin{abstract}
In this paper, we propose a Bayesian approach for affine auto-calibration. By the Bayesian approach, a posterior distribution for the affine camera parameters can be constructed, where also the prior knowledge can be taken into account. Moreover, due to the linearity of the affine camera model, the structure and translations can be analytically marginalised out from the posterior distribution, if certain prior distributions are assumed. The marginalisation reduces the dimensionality of the problem substantially that makes the MCMC methods better suitable for exploring the posterior of the intrinsic camera parameters. The experiments verify that the proposed approach is a versatile, statistically sound alternative for the existing affine auto-calibration methods.
\end{abstract}

\section{Introduction}

A central subject in computer vision is the reconstruction of $3 \mathrm{D}$ structure of the scene and camera parameters from multiple views that is also known as the structure-from-motion problem. An important related subproblem is the autocalibration problem, referred to determining the camera parameters without a known calibration object and, starting from [3, it has been widely researched in the computer vision community during the recent years (see [6, 4] for a review). Most of the auto-calibration literature assume the perspective projection camera model, but even auto-calibration methods for wide angle or non-central cameras have been proposed [9, 10].

This paper considers auto-calibration of an affine camera. The affine camera, introduced by [11, is an approximation of the projective camera model which is a generalisation of orthographic, weak perspective and para-perspective camera models and it is a tenable approximation if the viewing distance is large with respect to the depth of the object. The classical papers concerning structurefrom-motion using orthographic camera are e.g. 7] about affine reconstruction from two views and [17, where the factorisation approach was introduced. The auto-calibration of an affine camera has been earlier studied in [13]; an overview of the recent contributions on the field is given by [4]

The classical approaches for auto-calibration are based on first computing a (non-unique) projective, or affine in the case of an affine camera, reconstruction 
and the corresponding camera projection matrices. The projective, or affine, reconstruction is updated to metric by finding a rectifying transform that is determined by identifying the location of the absolute conic, or its dual, since it is the only invariant conic under rigid motion. Correspondingly, the affine auto-calibration method by Quan [13, is based on the invariance of the camera parameters and it also assumes an affine reconstruction as an input, computed e.g. by factorisation [17] that is updated to metric. Unification of affine and projective auto-calibration approaches has been discussed in [15].

This paper proposes a statistical method for automatic recovery of the affine camera parameters from uncalibrated image sequence. Unlike the auto-calibration algorithm proposed in [13, the proposed method computes the camera parameters directly from point correspondences without an intermediate affine reconstruction. As the solution for the auto-calibration, we shall have a posterior probability distribution for the camera parameters, where also the prior knowledge of the unknown parameters can be taken into account. Moreover, we will show that, because of linearity, the structure and translations can be analytically marginalised, together with the deviation parameter (see 2]), from the posterior distribution, as soon as suitable priors for them are assumed. Due to the marginalisation, the posterior distribution is more suitable to be explored by MCMC methods since dimensionality of the parameter space is notably reduced.

This paper is organised as follows. First the affine camera model and the principles of Bayesian approach are reviewed in Section 2 and 3 . Then the Bayesian approach for the auto-calibration is discussed in more detail and our solution for the problem is proposed. Experimental results are reported in Section 5] and conclusions are finally in Section 6 .

\section{Affine Camera Model}

The affine camera, introduced in [11, is obtained from the general $3 \times 4$ projection matrix $\mathbf{P}$ by setting the last row of to the form $\left(\begin{array}{llll}0 & 0 & 0 & 1\end{array}\right)$. The affine camera has thus 8 degrees of freedom and the corresponding (homogeneous) projection matrix is

$$
\mathbf{P}_{\mathrm{A}}=\left(\begin{array}{cccc}
m_{11} & m_{12} & m_{13} & t_{1} \\
m_{21} & m_{22} & m_{23} & t_{2} \\
0 & 0 & 0 & 1
\end{array}\right) .
$$

By denoting the top left $2 \times 3$ submatrix by $\mathbf{M}_{2 \times 3}$ and the top right 2 -vector by $\mathbf{t}$, and by using inhomogeneous coordinates, the projection equation is

$$
\left(\begin{array}{l}
x \\
y
\end{array}\right)=\mathbf{M}_{2 \times 3}\left(\begin{array}{l}
X \\
Y \\
Z
\end{array}\right)+\mathbf{t},
$$

where $\mathbf{t}$ is the image of the $3 \mathrm{D}$ origin or the image translation. 


\subsection{Parameterisation of the Affine Camera}

To obtain a representation for the camera parameters, the inhomogeneous projection matrix $\mathbf{M}_{2 \times 3}$ can be decomposed into the product $\mathbf{M}_{2 \times 3}=\mathbf{K R}$, where $\mathbf{K}$ is a $2 \times 2$ upper-triangular matrix and $\mathbf{R}$ a $2 \times 3$ matrix composed of two orthonormal rows, i.e.,

$$
\mathbf{M}_{2 \times 3}=\mathbf{K}_{2 \times 2} \mathbf{R}_{2 \times 3}=\left(\begin{array}{cc}
\alpha_{x} & s \\
0 & \alpha_{y}
\end{array}\right)\left(\begin{array}{l}
\mathbf{r}_{1} \\
\mathbf{r}_{2}
\end{array}\right) .
$$

This is known as the RQ decomposition, which can be computed as shown in [6] $\mathbf{K}_{2 \times 2}$ is the calibration matrix consisting at most three independent intrinsic camera parameters. $\mathbf{R}_{2 \times 3}$ contains two rows of the general rotation matrix in the $3 \mathrm{D}$ space and has thus 3 independent parameters. The three rotation and the two translation parameters form the set of extrinsic parameters.

\subsection{General Model}

Another way to parameterise the calibration matrix is

$$
\mathbf{K}_{2 \times 2}=k\left(\begin{array}{cc}
\xi_{a} & s_{a} \\
0 & 1
\end{array}\right),
$$

where $k$ is the scaling factor of the camera, $\xi_{a}$ is the aspect ratio of the camera and $s_{a}$ is the skew of the camera. This is the most general form of the affine camera calibration matrix, of which the special cases, weak perspective, scaled orthographic and orthographic projections, are obtained as follows.

\subsection{Weak Perspective Projection}

By assuming zero skew, we have the weak perspective model

$$
\mathbf{K}_{2 \times 2}=k\left(\begin{array}{cc}
\xi_{a} & 0 \\
0 & 1
\end{array}\right) \text {, }
$$

where the affine projection matrix has seven degrees of freedom (two intrinsic + five extrinsic parameters).

\subsection{Scaled Orthographic Projection}

The scaled orthographic model is obtained from the weak perspective model by setting equal scales for the $x$ and $y$ coordinate axes. The corresponding affine camera has thus six degrees of freedom and the calibration matrix is

$$
\mathbf{K}_{2 \times 2}=k\left(\begin{array}{ll}
1 & 0 \\
0 & 1
\end{array}\right) .
$$




\subsection{Orthographic Projection}

By setting the scaling factor $k$ of the previous calibration matrix equal to unity, the calibration matrix becomes $2 \times 2$ identity matrix and the orthographic projection model is obtained. This is the most simplified version of the affine camera having five degrees of freedom.

\section{Bayesian Approach}

In general, given a set of observed data samples $\mathcal{D}$ and a model $\mathcal{H}$, suppose there are unknown entities $\theta$ in the model. The idea of Bayesian approach is to create a probability distribution for all the unknown entities $\theta$. The distribution is known as the posterior probability distribution.

Following [5, suppose with fixed $\theta$ the data distribution $p(\mathcal{D} \mid \theta, \mathcal{H})$, denoted as the likelihood of the data, is known. Also suppose, that the distribution for $\theta$ in model $\mathcal{H}$ is known, often referred to as the prior distribution $p(\theta \mid \mathcal{H})$. Writing a product of these two distributions, a joint probability distribution

$$
p(\theta, \mathcal{D} \mid \mathcal{H})=p(\theta \mid \mathcal{H}) p(\mathcal{D} \mid \theta, \mathcal{H})
$$

for $\theta$ and $\mathcal{D}$ is obtained. Now according to the Bayes' rule, the posterior probability for parameters $\theta$ in model $\mathcal{H}$ with the given data samples $\mathcal{D}$, is

$$
p(\theta \mid \mathcal{D}, \mathcal{H})=\frac{p(\theta, \mathcal{D} \mid \mathcal{H})}{p(\mathcal{D} \mid \mathcal{H})}=\frac{p(\theta \mid \mathcal{H}) p(\mathcal{D} \mid \theta, \mathcal{H})}{p(\mathcal{D} \mid \mathcal{H})},
$$

where $p(\mathcal{D} \mid \mathcal{H})=\int_{\theta} p(\theta \mid \mathcal{H}) p(\mathcal{D} \mid \theta, \mathcal{H}) d \theta$ is a normalising constant and it does not depend on $\theta$. Thus, the unnormalised posterior probability distribution for $\theta$ is

$$
p(\theta \mid \mathcal{D}, \mathcal{H}) \propto p(\theta \mid \mathcal{H}) p(\mathcal{D} \mid \theta, \mathcal{H}) .
$$

\section{Bayesian Affine Auto-Calibration}

In this section we thus introduce the auto-calibration problem in the Bayesian framework (Sections 4.1 and 4.2). Our goal is to construct the probability distribution for the intrinsic camera parameters from a set of images. In Section 4.3. we discuss how to compute estimates from the posterior distribution.

\subsection{Statistical Model for the Data}

Now, suppose we have $N$ uncalibrated images with altogether $M$ separate points of which we observe projections but all the projections need not to be visible in all the views. Assuming 2D Gaussian noise model for the image point measurements $\mathbf{m} \equiv \mathcal{D}$, the joint probability distribution, or the likelihood, for all points $j$ in 
all images $i$ is written as

$$
p(\mathbf{m} \mid \theta, \mathcal{H}) \propto \prod_{i, j \mid \delta_{i j}=1} \sigma^{-2} \exp \left\{-\frac{1}{2 \sigma^{2}}\left(\mathbf{m}_{j}^{i}-\left(\mathbf{M}^{i} \mathbf{x}_{j}+\mathbf{t}^{i}\right)\right)^{\mathrm{T}}\left(\mathbf{m}_{j}^{i}-\left(\mathbf{M}^{i} \mathbf{x}_{j}+\mathbf{t}^{i}\right)\right) \delta_{i j}\right\}
$$

where $\delta_{i j}=1$ if point $j$ is visible in image $i$, otherwise it is zero. The model $\mathcal{H}$ here refers to the affine projection model (1) equipped with the 2D Gaussian measurement noise model. We here consider the case where the observed data contains distinct point projection coordinates in multiple views and we have established the correspondences between the point projections across the views. Thus, the unknown entities $\theta$ in the likelihood function are here both the camera parameters (both intrinsic and extrinsic) and the $3 \mathrm{D}$ reconstructions of the points, as well as the noise deviation $\sigma$.

\subsection{Posterior Distribution for the Camera Parameters}

Let our selected prior distribution for the unknown parameters $\theta$ be separable such that the posterior distribution is of the form

$$
p(\theta \mid \mathbf{m}, \mathcal{H}) \propto p(\sigma \mid \mathcal{H}) p(\mathbf{K} \mid \mathcal{H}) p(\mathbf{R} \mid \mathcal{H}) p(\mathbf{t} \mid \mathcal{H}) p(\mathbf{x} \mid \mathcal{H}) p(\mathbf{m} \mid \theta, \mathcal{H})
$$

In pure affine auto-calibration, we are not interested in the structure parameters $\mathbf{x}$, the camera extrinsic, motion parameters $\mathbf{R}, \mathbf{t}$, nor the noise deviation $\sigma$. In Bayesian framework, our complete ignorance of those parameters may be represented by marginalising them out from the posterior distribution. That is

$$
p(\mathbf{K} \mid \mathbf{m}, \mathcal{H})=\iiint \int p(\theta \mid \mathbf{m}, \mathcal{H}) d \mathbf{x} d \mathbf{R} d \mathbf{t} d \sigma
$$

where we have a posterior distribution over the intrinsic camera parameters. The posterior (8) represents a complete solution for the auto-calibration problem. Unfortunately, it is improbable that the integral would have an analytical solution. However, we observe that the affine camera model is linear with respect to the structure and translation parameters, hence, the following partial integral can be computed analytically

$$
p(\mathbf{K}, \mathbf{R} \mid \mathbf{m}, \mathcal{H})=\iiint p(\theta \mid \mathbf{m}, \mathcal{H}) d \mathbf{x} d \mathbf{t} d \sigma
$$

provided that we choose suitable priors for the marginalised variables, and fix the gauge freedom properly. For the deviation $\sigma$, we choose the Jeffrey's prior $p(\sigma \mid \mathcal{H})=\frac{1}{\sigma}$. Furthermore, if we select, for instance, uniform or Gaussian priors for $\mathbf{x}$ and $\mathbf{t}$, the (9) can be generally integrated analytically, excluding the nondegenerate motion or structure cases and incompletely fixed gauge freedom. Note however that the Bayesian approach could generally also handle degenerate cases by defining suitable priors or developing a model selection framework but they are beyond the scope of this paper. 
Before we are able to integrate (9), we must fix the gauge freedom, i.e., we must specify in which coordinate system we represent our variables. In structurefrom-motion, there is no absolute reference coordinate system, so we must define where and to which orientation we set the origin of our $3 \mathrm{D}$ coordinate system and also to which value we should define the global scale. Uncertainties are measured with respect to this selection, which should be taken kept in mind when interpreting the results. For algebraic simplicity, we select "a trivial gauge" by setting the $3 \mathrm{D}$ origin to the first point. Likewise, we select the first view as the reference view so that the rotations of the other cameras are measured with respect to the first camera, and, we set the scale of the first camera to unity.

In the case of uniform priors for the structure and translations, and after fixing the gauge as discussed, a tedious algebraic manipulation reveals that

$$
\begin{aligned}
p(\mathbf{K}, \mathbf{R} \mid \mathbf{m}, \mathcal{H}) \propto p(\mathbf{K} \mid \mathcal{H}) & p(\mathbf{R} \mid \mathcal{H})\left(\prod_{j=2}^{M}\left(\operatorname{det} \mathbf{C}_{j}\right)^{-\frac{1}{2}}\right)(\operatorname{det} \mathcal{C})^{-\frac{1}{2}} \\
& \times\left(\sum_{i, k} a_{i k}-\mathbf{b}^{\mathrm{T}} \mathcal{C}^{-1} \mathbf{b}\right)
\end{aligned}
$$

where

$$
\begin{aligned}
\mathbf{C}_{j} & =\sum_{i=1}^{N} \mathbf{M}^{i^{\mathrm{T}}} \mathbf{M}^{i} \delta_{i j}, a_{i k}=\sum_{j=1}^{M} \mathbf{m}_{j}^{i^{\mathrm{T}}}\left(\delta(i-k) \mathbf{I}-\delta(j-1) \mathbf{M}^{i} \mathbf{C}_{j}^{-1} \mathbf{M}^{k^{\mathrm{T}}}\right) \mathbf{m}_{j}^{k} \delta_{i j} \delta_{k j} \\
\mathcal{C} & =\left(\begin{array}{ccc}
\mathbf{C}_{11} & \cdots & \mathbf{C}_{1 N} \\
\vdots & \ddots & \vdots \\
\mathbf{C}_{N 1} & \cdots & \mathbf{C}_{N N}
\end{array}\right), \mathbf{C}_{i k}=\sum_{j=1}^{M}\left(\delta(i-k) \mathbf{I}-\delta(j-1) \mathbf{M}^{i} \mathbf{C}_{j}^{-1} \mathbf{M}^{k^{\mathrm{T}}}\right) \delta_{i j} \delta_{k j} \\
\mathbf{b} & =\left(\begin{array}{c}
\mathbf{b}_{1} \\
\vdots \\
\mathbf{b}_{N}
\end{array}\right), \quad \mathbf{b}_{k}=\sum_{i=1}^{N} \sum_{j=1}^{M}\left(\delta(i-k) \mathbf{I}-\delta(j-1) \mathbf{M}^{k} \mathbf{C}_{j}^{-1} \mathbf{M}^{i^{\mathrm{T}}}\right) \mathbf{m}_{j}^{i} \delta_{i j} \delta_{k j} .
\end{aligned}
$$

The posterior probability for the camera parameters will obtained by numerically evaluating

$$
p(\mathbf{K} \mid \mathbf{m}, \mathcal{H})=\int p(\mathbf{K}, \mathbf{R} \mid \mathbf{m}, \mathcal{H}) d \mathbf{R},
$$

where the integration is performed over the rotation parameters. The computation of estimates from this distribution is described in the following section.

\subsection{Computing Estimates from the Posterior}

The posterior distribution of the intrinsic camera parameters is a complete solution for the affine auto-calibration problem; from it we may compute estimates, i.e., a set of camera parameters that we could regard as the most descriptive candidates. We would also like to know the uncertainty of our estimates. At this step we have two obvious choices to proceed: we might compute the estimate 
that maximises the posterior (the MAP estimate) or the estimate that represents the conditional mean (the CM estimate) of the posterior distribution.

\subsection{MAP Estimate}

Computation of the maximum a posterior estimate is an optimisation problem. Let $\theta_{\mathbf{K}}$ represent the set of unknown intrinsic parameters of the affine cameras we have set unknown. Note that some parameters must be set known or invariant across the views, since otherwise the auto-calibration problem is not well defined. Since the rotations can not be marginalised from the posterior, we compute the MAP estimate of the joint posterior (10), i.e., we make the approximation

$$
\hat{\theta}_{\mathbf{K}, \mathrm{MAP}}=\arg \max _{\theta_{\mathbf{K}}} p(\mathbf{K} \mid \mathbf{m}, \mathcal{H}) \approx \arg \max _{\theta_{\mathbf{K}}}\left(\max _{\theta_{\mathbf{R}} \mid \theta_{\mathbf{K}}} p(\mathbf{K}, \mathbf{R} \mid \mathbf{m}, \mathcal{H})\right),
$$

where the maximum can be numerically computed by conventional nonlinear optimisation tools such as Levenberg-Marquardt. The uncertainty can be characterised by the covariance matrix of the estimated parameters. Here we approximate the posterior covariance by first making a Gaussian posterior approximation $p_{\text {approx }}(\mathbf{K}, \mathbf{R} \mid \mathbf{m}, \mathcal{H})$ around the maximum of $p(\mathbf{K}, \mathbf{R} \mid \mathbf{m}, \mathcal{H})$ by the Laplace's method $[8]$. Then we are able to marginalise the rotation parameters analytically, i.e., we compute

$$
\begin{aligned}
p(\mathbf{K} \mid \mathbf{m}, \mathcal{H}) & \approx \int p_{\text {approx }}(\mathbf{K}, \mathbf{R} \mid \mathbf{m}, \mathcal{H}) d \mathbf{R} \\
& \propto \exp \left(-\frac{1}{2}\left(\theta_{\mathbf{K}}-\hat{\theta}_{\mathbf{K}, \mathrm{MAP}}\right)^{\mathrm{T}} \tilde{\mathbf{C}}_{\theta_{\mathbf{K}}}^{-1}\left(\theta_{\mathbf{K}}-\hat{\theta}_{\mathbf{K}, \mathrm{MAP}}\right)\right),
\end{aligned}
$$

which is a Gaussian approximation over the intrinsic parameters only, hence, we can use the covariance matrix of this Gaussian approximation as our covariance matrix estimate for the intrinsic camera parameters.

\subsection{Estimate}

Another alternative for characterising the posterior, is to compute the conditional mean estimate, conditioned on the data, defined as

$$
\hat{\theta}_{\mathbf{K}, \mathrm{CM}}=\mathrm{E}\left\{\theta_{\mathbf{K}} \mid \mathbf{m}, \mathcal{H}\right\}=\iint \theta_{\mathbf{K}} p(\mathbf{K}, \mathbf{R} \mid \mathbf{m}, \mathcal{H}) d \theta_{\mathbf{K}} d \theta_{\mathbf{R}}
$$

which is an integration problem. Likewise, to characterise the uncertainty we would like to compute the conditional covariance $\mathbf{C}_{\theta_{\mathbf{K}}}$, defined as

$$
\operatorname{Cov}\left\{\theta_{\mathbf{K}} \mid \mathbf{m}, \mathcal{H}\right\}=\iint\left(\theta_{\mathbf{K}}-\hat{\theta}_{\mathbf{K}, \mathrm{CM}}\right)\left(\theta_{\mathbf{K}}-\hat{\theta}_{\mathbf{K}, \mathrm{CM}}\right)^{\mathrm{T}} p(\mathbf{K}, \mathbf{R} \mid \mathbf{m}, \mathcal{H}) d \theta_{\mathbf{K}} d \theta_{\mathbf{R}}
$$

Though the integrals cannot be calculated in closed form, they can be approximately evaluated by Markov chain Monte Carlo methods (see for instance 
[16] or [5]). By obtaining a reasonable amount of samples from the distribution, the conditional mean can be approximated as

$$
\hat{\theta}_{\mathbf{K}, \mathrm{CM}} \approx \frac{1}{n} \sum_{j=1}^{n} \theta_{\mathbf{K}}^{(j)},
$$

and the conditional covariance as

$$
\mathbf{C}_{\theta_{\mathbf{K}}} \approx \frac{1}{n-\operatorname{dim}\left(\theta_{\mathbf{K}}\right)} \sum_{j=1}^{n}\left(\theta_{\mathbf{K}}^{(j)}-\hat{\theta}_{\mathbf{K}, \mathrm{CM}}\right)\left(\theta_{\mathbf{K}}^{(j)}-\hat{\theta}_{\mathbf{K}, \mathrm{CM}}\right)^{\mathrm{T}},
$$

where $\theta_{\mathbf{K}}^{(1)} \ldots \theta_{\mathbf{K}}^{(n)}$ are assumed to be effectively independent samples obtained by $\mathrm{MCMC}$ simulation. Here the numerical computation of the CM estimate is rather convenient for the MCMC methods since the dimensionality of the parameter space is equal to $\operatorname{dim} \theta_{\mathbf{K}}+\operatorname{dim} \theta_{\mathbf{R}}$ which is typically substantially lower than the dimensionality of the original posterior (7).

\section{$5 \quad$ Experiments}

We experimented our auto-calibration method with two image sequences. The first set was a synthetic house sequence of 9 images, where the simulated images obey the perspective projection model. We identified and manually tracked 81 points from these images, so that the tracked points contained also missing data. Since the perspective effects were relatively strong in the projections, the role of this data set in our affine auto-calibration experiments is only illustrative.

For auto-calibration, we then used the scaled orthographic camera model and computed the MAP and CM estimates for the affine camera parameters from (12) and (16), respectively. For both rotation and scale parameters, we used the uniform prior distribution. The CM estimate was computed using the Metropolis-Hastings algorithm using a Gaussian proposal distribution. Due to gauge fixing, the scale of the first image was set to unity and similarly its rotation matrix was set to the identity matrix, and there were thus 8 scale parameters and $3 \times 8=24$ rotation parameters to be estimated, or, 32 parameters in total. The starting points for the optimisation and sampling were randomly generated.

To illustrate the result, we then recovered the 3D structure and translations, given the found estimates for the camera parameter values; the conditional estimates for structure and translations are computed as proposed in [1]. The MAP and $\mathrm{CM}$ estimates showed somewhat similar reconstructions here, as Fig. 1 1 illustrates. As it can be seen, the perspective effects can not been explained by the affine camera model due to which the angle between the two orthogonal walls is not straight as it was in the original simulated data.

As real data, we experimented the hotel image set, available at the CMU VASC image databas@1, that was also used in [12] and [14] (Fig. 22). In our

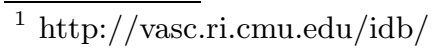


Fig. 1. Synthetic house data reconstructed after the recovery of the scaled orthographic camera parameters: (a) MAP estimate, front view; (a) CM estimate, front view; (c) MAP estimate, bottom view. (d) CM estimate, bottom view. The angle between the two wall planes is not straight, because the affine camera model is not able to model the perspective effects of the original data

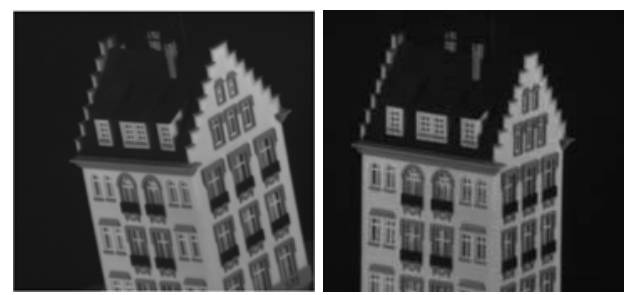

Fig. 2. Two examples from the Hotel model images. The set set is from CMU VASC image database

experiments, we used 10 of the 182 images, where 111 points were identified and tracked manually. All points were visible in all images, i.e., there was no missing data; the initial affine reconstruction in Quan's method [14] was computed using the factorisation algorithm [17]. For this set, we selected the weak perspective projection model, hence, there were 9 scales, 10 aspect ratios, plus 27 rotation parameters to be identified, i.e., 46 parameters in total.

The affine auto-calibration results for the Hotel set are illustrated in Fig. 3 Again, the MAP and CM estimates implied similar reconstruction, so only the

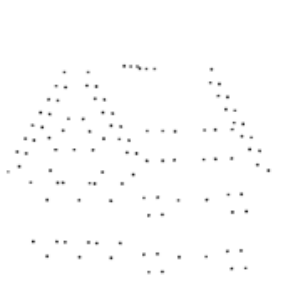

(a)

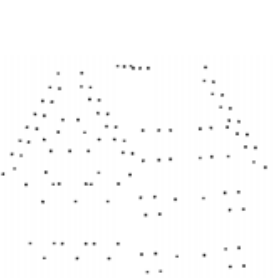

(b)

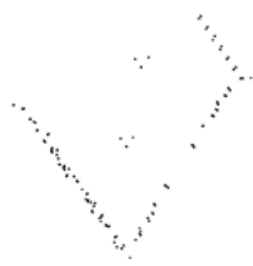

(c)

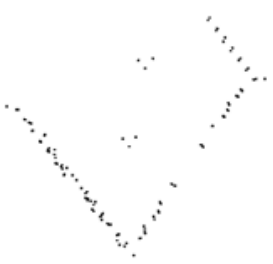

(d)

Fig. 3. Reconstruction of the Hotel set after determining the camera parameters by auto-calibration. (a) Quan auto-calibration, front view; (b) the MAP estimate, front view; (c) Quan auto-calibration, top view; (d) the MAP estimate, top view 
reconstruction corresponding the MAP estimate and the reconstruction corresponding to Quan's method are shown there. The MAP (and CM) estimate seems to be a bit more accurate than the Quan auto-calibration as the corner seen in the top view is more right.

\section{Summary and Conclusions}

This paper proposed a new statistical method auto-calibration from multiple images assuming an affine camera model. Like the earlier methods, the methods is based on the invariance of camera parameters, i.e., under the selected assumption of invariant parameters over the views, the affine camera parameters can be determined. As we used the Bayesian, statistical setting, available prior information of the camera parameters can be directly incorporated in the method. Moreover, the Bayesian approach for auto-calibration is well suitable for an affine camera model, since the uninteresting structure and translation variables, from the auto-calibration point of view, can be analytically marginalised from the posterior distribution under certain prior distribution assumptions. The marginalisation makes the MCMC methods better suitable for exploring the posterior distribution of unknown camera parameters as the large dimensionality of the structure-from-motion problem is substantially reduced. The experimental results validate that the proposed method is statistically sound method for affine auto-calibration, as it minimises statistical error in contrast to the earlier methods that minimise an algebraic error.

\section{References}

1. S. S. Brandt. Conditional solutions for the affine reconstruction of $N$ views. Image and Vision Computing, 2005. In press.

2. G. L. Bretthorst. Bayesian Spectrum Analysis and Parameter Estimation, volume 48 of Lecture Notes in Statistics. Springer, 1988.

3. O. Faugeras, Q. Luong, and S. Maybank. Camera self-calibration: Theory and experiments. In Proc. ECCV, pages 321-334, 1992.

4. O. Faugeras and Q.-T. Luong. Geometry of Multiple Images. The MIT Press, 2001.

5. A. Gelman, J. B. Carlin, H. S. Stern, and D. B. Rubin. Bayesian Data Analysis. Chapman and Hall/CRC, 2004.

6. R. Hartley and A. Zisserman. Multiple View Geometry in Computer Vision. Cambridge University Press, 2000.

7. J. Koenderink and A. van Doorn. Affine structure from motion. J. Opt. Soc. Am. A, 8(2):377-385, 1991.

8. D. MacKay. Information Theory, Inference and Learning Algorithms. Cambridge University Press, 2003.

9. B. Micusik and T. Pajdla. Estimation of omnidirectional camera model from epipolar geometry. In Proc. CVPR, volume 1, pages 485-490, 2003.

10. B. Micusik and T. Pajdla. Autocalibration \& 3 d reconstruction with non-central catadioptric cameras. In Proc. CVPR, volume 1, pages 58-65, 2004. 
11. J. L. Mundy and A. Zisserman. Geometric Invariance in Computer Vision. MIT Press, Cambridge, Massachusetts, 1992.

12. C. J. Poelman and T. Kanade. A paraperspective factorization method for shape and motion recovery. In Proc. ECCV, pages 97-108, 1994.

13. L. Quan. Self-calibration of an affine camera from multiple views. Int. J. Comput. Vis., 19(1):93-105, 1996.

14. L. Quan and T. Kanade. A factorization method for affine structure from line correspondences. In Proc. CVPR, pages 803-808, 1996.

15. L. Quan and B. Triggs. A unification of autocalibration methods. In Proc. ACCV, 2000.

16. C. P. Robert and G. Casella. Monte Carlo Statistical Methods. Springer, 1999.

17. C. Tomasi and T. Kanade. Shape and motion form image streams under orthography: A factorization approach. Int. J. Comput. Vis., 9(2):137-154, 1992. 